\title{
Food-borne outbreak of norovirus infection in a French military parachuting unit, April 2011
}

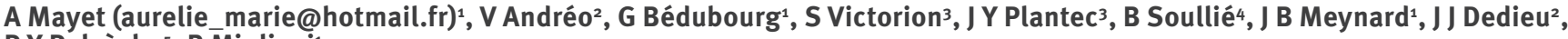 \\ P Y Polvèche ${ }^{5}$, R Migliani ${ }^{1}$ \\ 1. Military Centre for Epidemiology and Public Health, Military Teaching Hospital Bégin, Saint Mandé, France \\ 2. Veterinary Department, Military Health Service, Toulouse, France \\ 3. School of Airborne Troops, Pau, France \\ 4. Medical Biology Service, Military Teaching Hospital R. Picqué, Bordeaux, France \\ 5. Regional Directorate of the Military Health Service, Bordeaux, France
}

Citation style for this article:

Mayet A, Andréo V, Bédubourg G, Victorion S, Plantec JY, Soullié B, Meynard JB, Dedieu JJ, Polvèche PY, Migliani R. Food-borne outbreak of norovirus infection in a French military parachuting unit, April 2011.

Euro Surveill. 2011;16(30):pii=19930. Available online: http://www.eurosurveillance.org/ViewArticle.aspx?Articleld=19930

On 13 April 2011 the medical service of a French military parachuting unit reported an outbreak of acute gastroenteritis involving 147 persons among the military personnel. Meals suspected to have caused the outbreak (pasta and some raw vegetables) were tested for norovirus by PCR. The same norovirus (genogroup I) was found in some of the food items consumed by the cases and in a cook who prepared the meals.

On 13 April 2011, the medical service of a French military parachuting unit reported to the French military epidemiological centre an outbreak of acute gastroenteritis involving more than 100 persons who had fallen ill on 12 and 13 April 2011. The clinical symptoms reported by the patients were fever, vomiting and diarrhoea. Food from the military base canteen was suspected to be a potential vehicle of infection. An investigation was initiated on 14 April to describe the extent of the outbreak, to identify the causative pathogen, the mode of transmission and the source of infection, and to implement infection control measures. We report the results of this investigation as a reminder that food-borne disease outbreaks can present an important problem, particularly in settings such as military establishments where people live in close proximity and share the same facilities $[1,2]$.

\section{Methods}

We collected information on demographics, clinical symptoms and disease onset from personnel with acute gastroenteritis among the staff present on site at the time of the investigation $(n=295)$. A case was defined as a member of the military unit staff who presented at least one measurable symptom of the following: diarrhoea defined by three or more liquid stools in 24 hours, vomiting, and oral temperature of $\geq 38^{\circ} \mathrm{C}$ between 11 and 15 April 2011. In order to test the hypothesis that food items served in the canteen were the vehicles of infection, we conducted a casecontrol study which only targeted the meals served between 11 and 12 April because the unit had been closed on 9 and 10 April. Because part of the staff in the unit was not present at the time of the investigation, a retrospective cohort study was not possible. On 14 April, we distributed a standardised questionnaire to all members of the staff present in the unit. These included cases but also other staff members who did not experience any symptoms and who were therefore considered as controls. This questionnaire collected information on dates of onset of illness, symptoms and types of food consumed. Participation was voluntary and anonymous. In parallel, another investigation was initiated on 13 April by the veterinary department of the Military Health Service. This investigation consisted in the inspection of the catering process and the verification of hygiene procedures in place. Data were analysed using Stata (Stata Statistical Software: Release 9, Texas). The statistical strength of the associations was estimated by odds ratios (OR) with $95 \%$ confidence intervals $(\mathrm{Cl})$. In addition to the bivariate analysis, a multivariate analysis was performed using logistic regression.

At French military base canteens, meal items are routinely sampled and samples are kept for five days. We tested for norovirus the water of the drinking fountains and the food items served and sampled in the canteen on 11 and 12 April, which were suspected to be associated with the outbreak following the analytical study. The extracted RNA was tested for norovirus by real-time RT-PCR [3]. Pasta was tested by culture for Bacillus cereus which was initially suspected to have caused the outbreak by the physicians who treated the cases. In addition, water from the drinking fountain was tested by culture for coliform germs. For logistical reasons, no samples were requested from the cases, apart from a cook who had prepared the meals and who had fallen ill before the outbreak. The stool sample from the cook was tested for norovirus by PCR as described [3]. 


\section{Results}

During the investigation, 295 individuals (all staff present in the unit at the time of the investigation) were interrogated ( $58 \%$ of 466 persons who had eaten in the canteen on 11 and 12 April). The information regarding the total number of people who had eaten in the canteen on 11 and 12 April was obtained from the sales register of the canteen. Among 169 individuals who reported symptoms, 147 met the case definition. This corresponds to 147 cases of 295 persons who were interrogated. The remaining persons were used as controls in our study and this results in approximately one control per case. Symptoms reported by the patients included vomiting ( $85 \%)$, diarrhoea $(79 \%)$ with a mean of four liquid stools a day, abdominal pain (91\%), nausea $(87 \%)$ and fever (73\%). An oral temperature of $\geq 38^{\circ} \mathrm{C}$ was objectively measured in three patients. Date of symptom onset was known only for 138 cases of the 147 persons who met the case definition. The first cases occurred on 11 April in the evening and the last in the morning of 14 April, with an epidemic peak during the night of 12 April (Figure). The first cases included the cook who prepared cold dishes in the canteen on 11 and 12 April and who experienced abdominal pain and nausea starting with 11 April.

The analytical study performed on 69 food items used in four meals, showed a significant association between the occurrence of illness and the consumption of salad (OR: $2.1 ; 95 \% \mathrm{Cl}: 1.0-4.4 ; \mathrm{p}=0.03$ ) and raw vegetables (OR: $2.1 ; 95 \% \mathrm{Cl}: 1.1-3.8 ; p=0.01)$ which were prepared by the ill cook and served on 11 April at lunch and dinner. Other associations were found with water taken from a drinking fountain for the dinner of 11 April (OR: 2.6; 95\% Cl: 1.0-7.0; $p=0.03$ ) and pasta served on 12 April dinner (OR: 2.7; 95\% Cl: 1.1-7.1; $\mathrm{p}=0.01$ ). All these statistical links disappeared in the multivariate analysis, which was not surprising due to the high number of food items tested.

As this outbreak involved a large number of cases (including a majority who occurred during a parachuting training on 12 April at night and some cases among physicians of the medical service), no samples were taken from any of the cases. However, a stool sample was tested from the ill cook and was found positive for norovirus genogroup I by PCR. Following the results of the analytical study, uncooked vegetables served on 11 April were tested for norovirus. The same genogroup I norovirus was found in carrots and salad served at lunch, and in the tomatoes served at dinner. No further genotyping was performed. Among the cases, $72 \%$ had eaten at least one of these food items. The culture of Bacillus cereus from pasta served on 12 April was negative. The pasta was also negative for norovirus. Analyses performed on the water from the drinking fountains did not find noroviruses or any other pathogen but retrieved coliform germs. The investigation performed by the veterinary services also revealed some dysfunction in the cold chain concerning the preparation of the cold starters and in the maintenance of the drinking fountains. Water fountains were closed until disinfection and recommendations for hygiene were given to the company responsible for catering.

\section{Discussion and conclusion}

This norovirus-related food-borne disease outbreak involving 147 cases occurred during a parachuting exercise on the night of 12 April and affected significantly the activities of the military unit. It is interesting to note that another outbreak of acute gastroenteritis occurred between 10 and 12 April among residents of a retirement home in the same geographical area, in which the same cook involved in the outbreak in the military unit prepared food on 9 and 10 April. However, the outbreak in the nursing home was only suspected after interrogation of the ill cook; it had not been reported to the health authorities and consequently, it had not been investigated, but it is likely that it was also caused by norovirus considering that around 50\% of acute gastroenteritis outbreaks in industrialised countries are related to this agent [4]. Other norovirus outbreaks related to raw vegetables have been described in the past in other military units $[5,6]$. The episode described here illustrates once more that food-borne disease outbreaks can easily occur in such settings and stricter hygiene measures may need to be considered.

Despite the fact that no samples were taken from cases, the presence of genogroup I norovirus in the cook who had fallen ill 24 hours before the outbreak and in some food items which he prepared, implicate him as the source of the outbreak. As the norovirus incubation period ranges from 6 to 48 hours [7] we may assume the following hypothetical sequence of events: likely contamination of the cook at the retirement home, contamination of the food items prepared by the cook for lunch and dinner on 11 April in the military unit, the occurrence of the first cases among military staff on 11 April in the evening (six hours after the first assumed

\section{FIGURE}

Acute gastroenteritis outbreak due to norovirus infection in a French military parachuting unit, April $2011(\mathrm{n}=138$ cases $^{\mathrm{a}}$ )

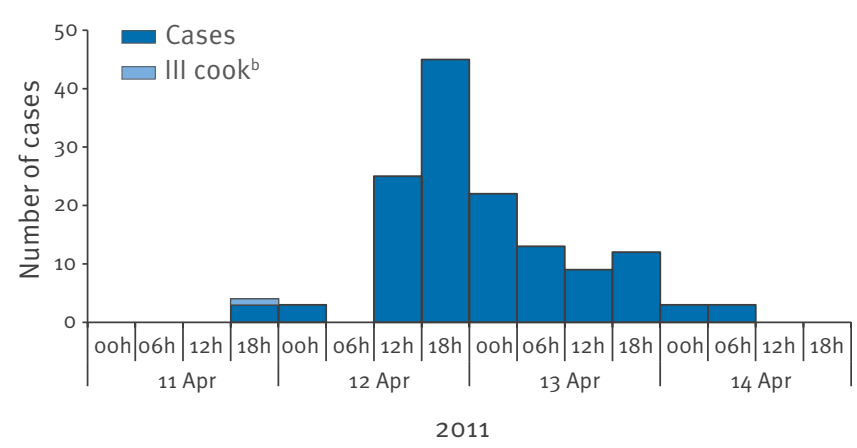

${ }^{a}$ With known date of symptom onset

b The cook presented only subjective symptoms and did not meet the case definition, but was added to the curve for a better understanding of the outbreak. 
food contamination), and the epidemic peak during the night of 12 April (24-36 hours after supposed food contamination). However, considering the date of symptom onset of the cook, it may also be possible that he contaminated both places.

The symptoms reported by the patients, particularly the fever, are compatible with a norovirus infection [7]. The infectious dose of norovirus is known to be low; therefore many food items could have been contaminated by one person only [4]. Due to the fact that in military settings people live in close proximity and share the same facilities, secondary human-to-human transmission of norovirus appears possible, which may account for the large number of cases involved in this outbreak [8]. Statistical associations observed between consumption of water and symptoms could account for a human-to-human contamination via the drinking fountains shared by the cases.

The fact that the investigation occurred 48 hours after the outbreak and that only $58 \%$ of the staff present during the episode could be interrogated, may have resulted in a lack of power of the analytical study and may also be a source of potential bias. This could explain the fact that not all dishes prepared by the ill cook were significantly associated with illness.

The fact that norovirus was detected in a non-diarrhoeic stool sample from the cook who presented only few symptoms, underlines the importance of testing samples from cases even when they are non symptomatic. Recent laboratory techniques permit the detection of norovirus from faeces up to approximately seven days following the infection $[9,10]$. Therefore physicians should be encouraged to collect samples from patients even if the outbreak is over.

In conclusion, this investigation demonstrates that food-borne disease outbreaks may have certain impact on the operational activities of settings such as military units but, on the other hand, the operational context may perturb the investigation and create difficulties in the identification of the vehicle of infection.

References

1. Grotto I, Mandel Y, Ashkenazi I, Shemer J. [Epidemiological characteristics of outbreaks of diarrhea and food poisoning in the Israel Defense Forces in the years 1978-1995]. Harefuah. 1997;133(7-8):255-64, 336. Hebrew.

2. Mayet A, Chai N, Bougherra C, Rous V, Lagathu G, Duron S, et al. Clostridium perfringens related food poisoning outbreak among fire soldiers in Paris: investigation is suitable despite a low population size. Med Armees. 2009;37(4):373-6. French.

3. da Silva AK, Le Saux IC, Parnaudeau S, Pommepuy M, Elimelech M, Le Guyader FS. Evaluation of removal of noroviruses during wastewater treatment, using real-time reverse transcription-PCR: different behaviors of genogroups I and II. Appl Environ Microbiol. 2007;73(24):7891-7.

4. Teunis PF, Moe CL, Liu P, Miller SE, Lindesmith L, Baric RS, et al. Norwalk virus: how infectious is it?. J Med Virol. 2008;80(8):1468-76.

5. Wadl M, Scherer K, Nielsen S, Diedrich S, Ellerbroek L, Frank $C$, et al. Food-borne norovirus-outbreak at a military base, Germany, 2009. BMC Infect Dis. 2010;10:30.
6. Grotto I, Huerta M, Balicer RD, Halperin T, Cohen D, Orr N, et al. An outbreak of norovirus gastroenteritis on an Israeli military base. Infection. 2004;32(6):339-43.

7. Patel MM, Hall AJ, Vinjé J, Parashar UD. Noroviruses: a comprehensive review. J Clin Virol. 2009;44(1):1-8.

8. Kaplan JE, Schonberger LB, Varano G, Jackman N, Bied J, Gary GW. An outbreak of acute nonbacterial gastroenteritis in a nursing home. Demonstration of person-to-person transmission by temporal clustering of cases. Am J Epidemiol. 1982;116(6):940-8.

9. Atmar RL, Opekun AR, Gilger MA, Estes MK, Crawford SE, Neill $\mathrm{FH}$, et al. Norwalk virus shedding after experimental human infection. Emerg Infect Dis. 2008;14(10):1553-7.

10. Kirby A, Gurgel RQ, Dove W, Vieira SCF, Cunliffe NA, Cuevas LE. An evaluation of the RIDASCREEN and IDEIA enzyme immunoassays and the RIDAQUICK immunochromatographic test for the detection of norovirus in faecal specimens. J Clin Virol. 2010;49(4):254-7. 